\title{
Relatively Low Prevalence of Peripheral and Placental Plasmodium Infection at Delivery in Bangui, Central African Republic
}

\author{
Alexandre Manirakiza, ${ }^{1,2}$ Eugène Serdouma, ${ }^{3,4}$ Djibrine Djalle, ${ }^{1}$ Georges Soula, ${ }^{2}$ \\ Remi Laganier, ${ }^{1}$ Nestor Madji, ${ }^{3}$ Methode Moyen, ${ }^{3}$ Alain Le Faou, ${ }^{5}$ and Jean Delmont ${ }^{2}$ \\ ${ }^{1}$ Institut Pasteur de Bangui, Avenue Pasteur, P.O. Box 923, Bangui, Central African Republic \\ ${ }^{2}$ Centre de Formation et de Recherche en Médecine et Santé Tropicales, Faculté de Médecine Nord, Boulevard Dramard, \\ 13015 Marseille, France \\ ${ }^{3}$ Reproductive Health and Malaria Program Division, Ministry of Public Health, Population and AIDS Control, Bangui, \\ P.O. Box 883, Bangui, Central African Republic \\ ${ }^{4}$ Faculty of Health Sciences, University of Bangui, P.O. Box 1383, Central African Republic \\ ${ }^{5}$ Hôpital de Brabois Adultes, CHU de Nancy, 54511 Vandoeuvre-lès-Nancy Cedex, France
}

Correspondence should be addressed to Alexandre Manirakiza, amanirak@yahoo.fr

Received 23 August 2011; Accepted 27 September 2011

Academic Editor: Jean-Paul Gonzalez

Copyright (C) 2011 Alexandre Manirakiza et al. This is an open access article distributed under the Creative Commons Attribution License, which permits unrestricted use, distribution, and reproduction in any medium, provided the original work is properly cited.

Introduction. The aim of this study was to estimate the prevalence of malaria among women giving birth in Bangui. Association between sociodemographic characteristics of those women and malaria, as well as prevention compliance (use of intermittent preventive treatment with sulfadoxine-pyrimethamine (IPTsp) and insecticide-treated bed nets (ITNs)), was analyzed. Methods. During September 2009, a survey was conducted on 328 women who gave birth at two main maternities of Bangui. Information was obtained by standardized questionnaire about sociodemographic criteria, IPTsp, other antimalarial treatment, and use of bet nets. Smears prepared from peripheral and placental blood were analysed for malaria parasites. Findings and Discussion. Positive results were found in $2.8 \%$ of thick peripheral blood smears and in $4.0 \%$ of placental slides. A proportion of $30.5 \%$ of the women had received at least two doses of IPTsp during the current pregnancy. Only a proportion of $42.4 \%$ of this study population had ITNs. Multigravid women were less likely to use IPTsp and ITNs. However, use of IPTsp was associated with personal income and secondary or university educational status. Hence, although this relatively prevalence was observed, more efforts are needed to implement IPTsp and ITNs, taking into account sociodemographic criteria.

\section{Introduction}

Every year, it is estimated that tens of thousands of pregnant women in malaria-endemic areas are infected with Plasmodium falciparum [1]. Frequently, placental infection occurs, owing to the accumulation of $P$. falciparum-infected erythrocytes in the intervillous space, despite the absence of parasites in peripheral blood [2]. The complications of malaria during pregnancy are maternal anaemia, preterm delivery, and low birth weight of newborns, which increase perinatal morbidity $[1,3,4]$. The World Health Organization (WHO) recommends intermittent preventive treatment with sulfadoxine-pyrimethamine (IPTsp) during pregnancy, with at least two doses after quickening (18-20 weeks) not more frequently than monthly, use of insecticide-treated bed nets (ITNs) and prompt treatment of clinical malaria [5]. Intermittent preventive treatment consists of delivering a curative treatment dose of an antimalarial at predefined intervals, regardless of the parasitological status of the woman, and the efficacy of this protocol has been demonstrated in a number of malaria-endemic countries [5-8].

Placental Plasmodium screening in the Central African Republic (CAR) in 1990 showed a rate of $37.1 \%$ in women who had been given chemoprophylaxis with chloroquine [9]. 
In 2006, the Ministry of Health of the CAR adopted and implemented the new WHO recommendations for malaria prevention during pregnancy. The aim of the study reported here was to estimate the prevalence of malaria in thick peripheral blood smears and placental blood from a sample of women who gave birth during September 2009 at two main maternities of Bangui, the capital of CAR. Secondly, we assessed the women's coverage rates with the three components of the WHO package [10] for malaria management during the current pregnancy and identify pregnant women characteristics associated with IPTsp and ITNs.

\section{Methods}

2.1. Study Setting. We conducted a cross-sectional study in the two main maternity clinics of Bangui, the Castors health Centre and the "Communautaire" Hospital, in September 2009. The geographic coordinates of Bangui is $7^{\circ} .00^{\prime}$ north and $21^{\circ} .00^{\prime}$ east. The climate is tropical, and rainfall peaks are observed from April to November, and temperature ranges from $19^{\circ} \mathrm{C}$ to $32^{\circ} \mathrm{C}$. The main parasite is Plasmodium falciparum, and malaria transmission is perennial with peaks during the rainy season, but no data on the intensity of malaria transmission (entomological inoculation rates) is available in CAR. Malaria represents more than $40 \%$ of morbidity in Bangui, as well as in other CAR areas [11].

The Castors Health Centre and the "Communautaire" Hospital provide antenatal and delivery services and have established programmes for the prevention of malaria and other infectious diseases, such as mother-to-child transmission of HIV infection. Each year, it is estimated that 12,000 women deliver at those centres, representing 70\% of all women who deliver in Bangui. All women are screened for malaria by microscopic analysis of $4 \%$ Giemsa-stained thick blood smears during visits to the antenatal clinics, performed either at the same health centre or at one of the national reference biomedical laboratories (the National Laboratory for Clinical Biology and Public Health and the Institut Pasteur de Bangui), depending on each woman's choice. Antimalarial treatment is prescribed on the basis of clinical symptoms if the woman cannot afford the laboratory fees immediately.

For asymptomatic women, IPTsp is given free of charge as directly observed therapy during the antenatal visit. Administration of two doses is recommended for HIV-negative women and three doses for HIV-positive women.

2.2. Study Population, Enrolment, and Data Collection. All women from whom we obtained written informed consent were eligible for the study immediately after delivery. A study midwife administered a standardized questionnaire to record sociodemographic data (age, residence area, literacy, number of gravidities, and monthly income), HIV serological status, intake of antimalarial medications (IPTsp and other antimalarial prescriptions), and bed net use.

Malaria prevalence of $30 \%$ at the time of childbirth was used as a proxy to calculate the sample size in this study. Thus, a number of 323 women was necessary assuming $90 \%$ power at 5\% significance level.
Because of lack of an ethical committee in the CAR, this project was reviewed and approved by an ad hoc scientific committee of the University of Bangui in charge of validating scientific study protocols in the CAR. The study was conducted under a collaborative agreement with the University of Marseille, France.

2.3. Laboratory Analyses. Peripheral venous and placental blood from each woman was used to prepare thick blood films. Placental blood was obtained as follows: immediately after delivery, the paracentric side of the maternal placenta was cleaned with sterile water and incised, and thick blood films were prepared from a droplet collected by aspiration through a 21-gauge needle attached to a $5-\mathrm{mL}$ syringe, as described previously $[12,13]$.

The thick smears were air-dried and stained with $4 \%$ Giemsa. At each of the two study sites, an experienced microscopist immediately examined the stained smears by light microscopy ( $\times 100$ oil immersion $)$ to detect asexual forms of P. falciparum malaria parasites. On peripheral blood slides, malaria parasites were counted in 200 leukocytes, and the parasite density per microlitre of blood was estimated as the number of parasites counted multiplied by 40 under the assumption of a leukocyte count of $8000 / \mu \mathrm{L}$ of blood. For both types of blood film, a result was considered to be negative if no parasites were detected per 200 leukocytes. Women with a positive peripheral blood result were given antimalarial treatment (either artemether-lumefantrine or quinine, depending on a clinical evaluation and individual tolerance).

All the slides were analysed twice at the Biomedical Laboratory of the Institut Pasteur de Bangui.

2.4. Data Analysis. Data were double-entered into EpiInfo software version 3.5.1, and the database was checked and data entry errors corrected with the EpiInfo software "data compare" utility for finding differences between two tables. Statistical analysis was conducted with Stata 8.0 and MedCalc v11.6.1. The association between sociodemographic criteria and malaria, use of IPTsp and ITNs was examined using the chi-squared test, and association between those variables was tested by calculating the odds ratios (ORs). The estimates were achieved at $95 \%$ interval confidence. Differences between proportions $\leq 5 \%$ were considered as significant.

\section{Results}

3.1. Study Population Characteristics. Overall, 328 pregnant women delivering at the two health centres were included in the study: $168(51.2 \%)$ at the Castors and $160(48.8 \%)$ at the "Communautaire" Hospital. A large proportion of them reside in Bangui city $(96.0 \%$ or $314 / 328)$. The mean age was 23 years (range, 14-39 years), and $33.8 \%$ were aged less than twenty years. A proportion of $33.0 \%$ (108/328) were primigravid. Most of those women do not have any personal monthly income $(57.9 \%)$, but the majority of them have at least secondary educational status.

HIV infection had been screened for 58.2\% (191/328) of the population, resulting an infection prevalence of $9.1 \%$ 


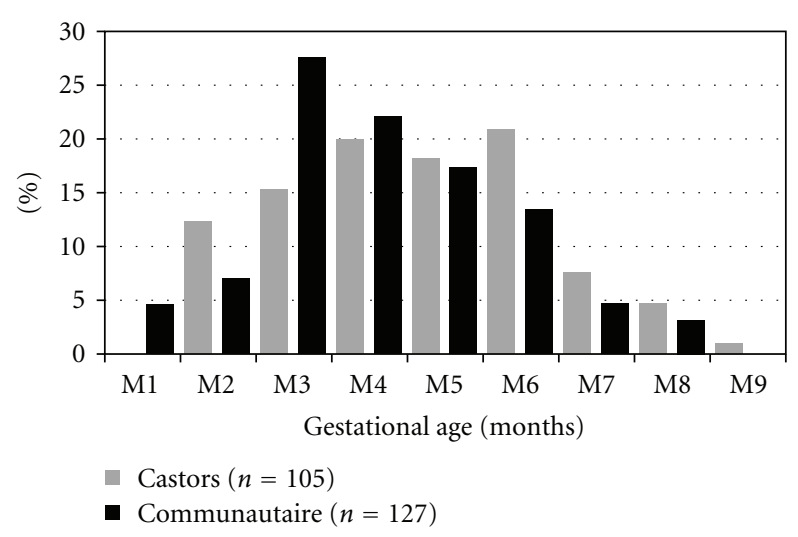

Figure 1: First antenatal clinic attendance according to gestational age, September 2009, Central African Republic.

(15/164; 27 HIV results could not be cross-checked on the antenatal clinic cards).

Sleeping daily under bed net was reported by $81.5 \%(95 \%$ CI, [77.3-85.7]) of the women, and $42.4 \%$ (95\% CI, [36.848.0]) had ITNs.

3.2. Attendance at Antenatal Clinics. At the two study sites, checking of antenatal clinic cards showed that $93.3 \%$ (95\% CI, [90.8-95.8]) of the women had presented at least one antenatal visit. Less than one fourth of the women $(24.1 \%$; 95\% CI, [19.3-28.9]) had attended an antenatal clinic during the first trimester of pregnancy, while the majority $(55.6 \%$; 95\% CI, [50.0-61.2]) had attended a clinic during the second trimester. Overall, 35.6\% (95\% CI, [31.1-40.1]) completed four antenatal visits. The distribution of first antenatal attendance according to gestational age at each study site is shown in Figure 1.

3.3. Any Antimalarial Treatment during the Pregnancy. Of the women who received IPTsp, 75.4\% (95\% CI, [69.181.8]) were given curative prescriptions of other antimalarial drugs, independently of the timing of IPTsp doses (Figure 2). The antenatal clinic cards of 182 women (55.5\%; 95\% CI, [50.1-60.1]) showed a history of at least one curative treatment for malaria. Of these women, $27.0 \%$ had been prescribed an antimalarial drug two or three times. Although 228 antimalarial prescriptions were recorded on antenatal clinic cards during the current pregnancy, only 56 laboratory results were positive out of the total 73 blood smears analysed. The antimalarial drugs prescribed were quinine (66.7\% or $152 / 228 ; 95 \%$ CI, [60.6-72.8]), artemisinin-based combinations $(15.5 \%$; 95\% CI, [10.7-20.1]) and artemisinin monotherapy (18.0\%; 95\% CI, [13.0-23.0]).

At least one dose of IPTsp was recorded for 54.6\% (95\% CI, [49.2-60.0]) of our study population; only 30.5\% (95\% CI, [23.8-37.2]) had received at least two doses. Most of these women (78.2\%; 95\% CI, [72.2-84.3]) had received the first IPTsp dose between the fourth and seventh months of pregnancy; however, $11.7 \%$ (95\% CI, [7.0-16.4]) had

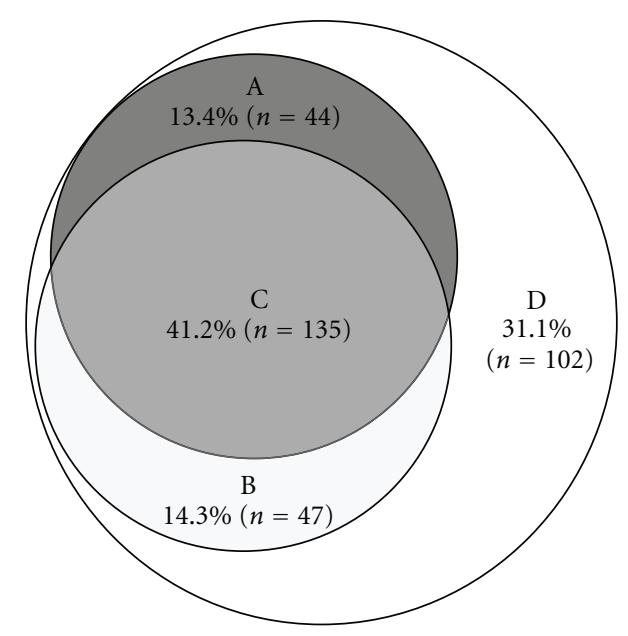
A: IPTsp only
B: Other antimalarial only
C: IPTsp and other antimalarial
D: No history of antimalarial

FIGURE 2: Distribution of antimalarial (IPTsp or any other antimalarial) use during pregnancy, September 2009, Central African Republic. IPTsp: intermittent preventive treatment with sulfadoxine-pyrimethamine.

received the first dose during the first trimester. Details of IPTsp administration are shown in Figure 3.

3.4. Relation between Sociodemographic Characteristics and the Compliance to Malaria Prevention. Multigravid women, were less likely to use two doses of IPTsp (OR $=0.14 ; 95 \%$ CI, [0.08-0.24], $P<0.0001)$ and ITNs (OR $=0.16$; 95\% CI, [0.10-0.28], $P<0.001)$ compared to primigravid women. Use of IPTsp (two doses) was associated with lucrative activities $(\mathrm{OR}=4.20$; [2.55-6.92], $P<0.0001)$ and secondary or university educational status $(\mathrm{OR}=2.22$; 95\% CI, [1.333.72], $P=0.002)$. Women with secondary or university educational status were also likely to use INTs $(\mathrm{OR}=1.90$; 95\% CI, [1.20-3.01], $P=0.01)$. Details on association analysis between sociodemographic characteristics and those preventive tools use are shown in Table 1.

3.5. Results of Blood Smear Examinations. Overall, peripheral blood $P$. falciparum infection at delivery was found in $2.8 \%$ (95\% CI, [1.0-4.6]) of peripheral blood and in $4.0 \%$ (95\% CI, [2.0-6.0]) of placental blood. Of the women with placental malaria, $77.0 \%$ (10/13) declared not using any bed net and 53.8\% (7/13) had not taken any antimalarial drug during pregnancy.

HIV serological status had no impact on these findings. IPTsp and ITNs use was found not to be associated with the women sociodemographical characteristics. Moreover, there is no statistically significant association between these laboratory findings and those characteristics. 
TABLE 1: Association between some sociodemographic characteristics and the compliance to intermittent preventive treatment with sulfadoxine-pyrimethamine (IPTsp) and insecticide-treated nets (INTs) in Bangui, September 2009, Central African Republic.

\begin{tabular}{|c|c|c|c|c|c|c|c|}
\hline \multirow[b]{2}{*}{ Criteria } & \multirow[b]{2}{*}{$n$} & \multicolumn{3}{|c|}{$\geq 2$ doses of IPTsp } & \multicolumn{3}{|c|}{ Use of ITNs } \\
\hline & & $\%$ & OR [95\% CI] & $P$-value & $\%$ & OR [95\% CI] & $P$-value \\
\hline \multicolumn{8}{|l|}{ Age (years) } \\
\hline$<20$ & 111 & 36.9 & 1 & NA & 48.6 & 1 & NA \\
\hline $20-24$ & 105 & 27.6 & $0.65[0.36-1.15]$ & 0.18 & 40.0 & $0.70[0.41-1.20]$ & 0.25 \\
\hline $25-29$ & 74 & 27.0 & $0.63[0.33-1.20]$ & 0.21 & 29.7 & $0.44[0.23-0.83]$ & 0.01 \\
\hline$\geq 30$ & 38 & 26.3 & $0.60[0.26-1.38]$ & 0.32 & 55.3 & $1.30[0.62-2.73]$ & 0.6 \\
\hline \multicolumn{8}{|l|}{ Gravidity } \\
\hline 1 & 108 & 58.3 & 1 & NA & 70.4 & 1 & NA \\
\hline$\geq 2$ & 220 & 16.8 & $0.14[0.08-0.24]$ & $<0.0001$ & 28.6 & $0.16[0.10-0.28]$ & $<0.001$ \\
\hline \multicolumn{8}{|l|}{ Monthly income } \\
\hline No income & 190 & 17.9 & 1 & NA & 46.3 & 1 & NA \\
\hline Salary or other personal income & 138 & 47.8 & $4.20[2.55-6.92]$ & $<0.0001$ & 37.0 & $0.67[0.43-1.06]$ & 0.11 \\
\hline \multicolumn{8}{|l|}{ Educational status } \\
\hline None or primary & 130 & 20.8 & 1 & NA & 33.1 & 1 & NA \\
\hline Secondary or university & 198 & 36.9 & $2.22[1.33-3.72]$ & 0.002 & 49.5 & $1.90[1.20-3.01]$ & 0.01 \\
\hline
\end{tabular}

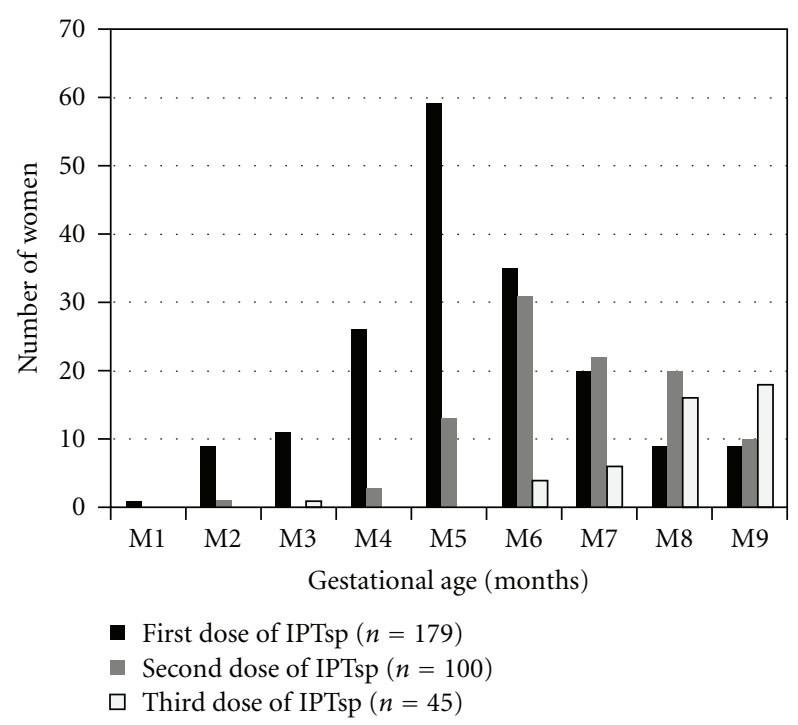

FIGURE 3: Distribution of intermittent preventive treatment with sulfadoxine-pyrimethamine (IPTsp) doses by gestational age, September 2009, Central African Republic.

\section{Discussion}

The prevalence of malaria among pregnant women in the CAR $(2.8 \%$ of thick peripheral blood smears and $4.0 \%$ of placental slides) was lower than in other areas of intense malaria transmission, such as Gabon, where the rates were $34.4 \%$ in maternal blood and $53.6 \%$ in placental blood films [6]. A recent review [14] of randomized clinical trials and surveys on the efficacy of IPT showed overall placentapositive rates $\geq 10 \%$. Falade and coauthors [7] in Nigeria reported that the prevalence of placental parasitaemia was $10.5 \%$ in women given IPTsp and $17 \%$ in those with no chemoprophylaxis.

Three years after initiation of IPTsp in CAR, coverage with at least one dose of IPTsp was slightly more than $50 \%$. Although WHO recommends two doses IPTsp for $\geq 80 \%$ of pregnant women [15], our estimate in this study was $30.5 \%$. The low prevalence of placental malaria in our study is therefore probably due to the combination of IPTsp, other antimalarial drug, and use of ITNs. Hence, the relatively low prevalence of malaria at delivery is not surprising in Bangui. Indeed, a similar finding of malaria prevalence at the time of delivery is observed in Côte d'Ivoire, where Vanga-Bosson and coauthors report a prevalence of $4.8 \%$ of placental malaria, in an area where IPTsp coverage rate ( $\geq 2$ doses) does not exceed 50\% [16] and, in Thailand, where proportion of positive results for $P$. falciparum in maternal blood and in placental blood were estimated at $3.0 \%$ and $3.8 \%$, respectively [17]. In Thailand, the authors report that this relative lower prevalence was due to antimalarial treatment with artemisinin derivatives. In our study, most of women were prescribed quinine or artemisinin-based combinations during the pregnancy. Indeed, those antimalarial drugs are actually the most efficacious on malaria [18].

Otherwise, our findings show that more than $80 \%$ of the women slept under a bed net. The efficacy of bed nets for preventing malaria is indisputable. Indeed, use of ITNs was found to reduce the incidence of uncomplicated malarial episodes in areas of stable malaria by $50 \%$ in comparison with no nets use and by $39 \%$ in comparison with untreated nets use [15].

In our study, the lower proportion of use of IPTsp and ITNs in multigravid women could be due to less health conscious of those women of their pregnancy. Inversely, 
the relative high proportion of use of IPTsp and ITNs in women with high level of education is due to the fact that higher educational status implies more health consciousness and is a factor influencing assimilation of health education programmes. Similar findings were reported in Cameroon and in Malawi $[19,20]$. Women with salary or other personal income are also likely to be compliant with IPTsp and ITNs, possibly because they are able to afford health care fees.

The main limitation of our study is the only use of microscopic examination of blood smears. Even if, the microscopic analysis remains the standard detection of Plasmodium [21], submicroscopic infections are common during pregnancy $[21,22]$. Hence, molecular methods (polymerase chain reaction or PCR) and rapid diagnostic tests (RDTs) provide finding approximately twice as many infections as microscopy [23-25]. However, determination of the possible impact of these submicroscopic infections to poor birth outcomes and maternal health is critical [26], and PCR is not feasible routinely [27]. To this end, microscopic examination to detect these infections is still essential, and implementation of RDTs use is challenging $[21,28]$.

\section{Conclusion}

Our results indicate that, although the recommended coverage rates of pregnant women with IPTsp and ITNs are not reached in Bangui, the prevalence of the main indicator of infection, placental malaria, is relatively low. The widespread presumptive prescription and consumption of antimalarial agents could indisputably be the cause of clearance of the existing peripheral and placental Plasmodium infection and decreased the risk of new infections over the pregnancy period. Indeed, symptoms suggestive of malaria are very frequent among pregnant women attending antenatal clinics, thus implying frequently unnecessary large use of antimalarial drugs [29]. For this purpose, strengthening national malaria control activities, taking into account prompt laboratory diagnosis and sociodemographic particularities, should contribute to the achievement of high coverage rate with the WHO preventive package components. Otherwise, cohort studies are needed to assess the real efficacy of IPTsp in preventing malaria during pregnancy.

\section{Authors' Contributions}

A. Manirakiza and J. Delmont conceived the study. Field data and blood samples analysis were achieved by A. Manirakiza, M. Moyen, D. Djalle, N. Madji and R. Laganier. Data analysis and interpretation were achieved by A. Manirakiza, J. Delmont, E. Serdouma, G. Soula, and Alain Le Faou. This draft was written by A. Manirakiza and J. Delmont. All authors read and approved this paper.

\section{Conflict of Interests}

The authors declare that they have no competing interests.

\section{Acknowledgments}

The authors are grateful to all the women who participated in this study. They also thank the staffs of the maternity clinics of Castors health center and "Communautaire" hospital in Bangui, where the study was conducted, which kindly collaborated in data collection. Many thanks also to Dr Elisabeth Heseltine (http://www.communicationinscience.com/) and to Dr Mirdad Kazanji for critical reading of the manuscript. This study received financial support from the "Service de la Coopération et d'Action Culturelle" of the "Ministère français des Affaires étrangères" and from Institut Pasteur de Bangui.

\section{References}

[1] M. Desai, F. O. ter Kuile, F. Nosten et al., "Epidemiology and burden of malaria in pregnancy," The Lancet Infectious Diseases, vol. 7, no. 2, pp. 93-104, 2007.

[2] M. Fried and P. E. Duffy, "Maternal malaria and parasite adhesion," Journal of Molecular Medicine, vol. 76, no. 3-4, pp. 162-171, 1998.

[3] R. W. Steketee, B. L. Nahlen, M. E. Parise, and C. Menendez, "The burden of malaria in pregnancy in malaria-endemic areas," The American Journal of Tropical Medicine and Hygiene, vol. 64, no. 1-2, pp. 28-35, 2001.

[4] J. E. Yartey, "Malaria in pregnancy: access to effective interventions in Africa," International Journal of Gynecology and $\mathrm{Ob}$ stetrics, vol. 94, no. 3, pp. 364-373, 2006.

[5] P. L. Olliaro, H. Delenne, M. Cisse et al., "Implementation of intermittent preventive treatment in pregnancy with sulphadoxine/pyrimethamine (IPTp-SP) at a district health centre in rural Senegal," Malaria Journal, vol. 7, article 234, 2008.

[6] M. K. Bouyou-Akotet, S. Nzenze-Afene, E. B. Ngoungou et al., "Burden of malaria during pregnancy at the time of IPTp/SP implementation in Gabon," The American Journal of Tropical Medicine and Hygiene, vol. 82, no. 2, pp. 202-209, 2010.

[7] C. O. Falade, B. O. Yusuf, F. F. Fadero, O. A. Mokuolu, D. H. Hamer, and L. A. Salako, "Intermittent preventive treatment with sulphadoxine-pyrimethamine is effective in preventing maternal and placental malaria in Ibadan, south-western Nigeria," Malaria Journal, vol. 6, article 88, 2007.

[8] P. Garner and A. M. Gülmezoglu, "Drugs for preventing malaria in pregnant women," Cochrane Database of Systematic Reviews, no. 4, p. CD000169, 2006.

[9] J. Testa, J. Awodabon, N. Lagarde, T. Olivier, and J. Delmont, "Interest in placental apposition as an epidemiological marker for malaria," Bulletin de la Societe de Pathologie Exotique, vol. 84, no. 5, pp. 473-479, 1991.

[10] WHO, A Strategic Framework for Malaria Prevention and Control During Pregnancy in the African Region, World Health Organization, Brazzaville, The Republic of the Congo, 2nd edition, 2004, http://whqlibdoc.who.int/afro/2004/AFR_MAL_ 04.01.pdf.

[11] Ministère de la Santé de la Population et de la lutte contre le VIH/SIDA, "Statistiques nationales d'information sanitaire (SNIS) 2009," Rapport annuel, Ministère de la Santé de la Population et de la lutte contre le VIH/SIDA, Bangui, Central African Republic, 2009.

[12] A. Sowunmi, A. E. J. Abohweyere, J. A. Akindele, A. O. Ilesanmi, C. O. Falade, and A. M. J. Oduola, "Comparison of the incision and aspiration methods for the diagnosis of 
placental malaria infection," Journal of Obstetrics and Gynaecology, vol. 16, no. 5, pp. 316-320, 1996.

[13] S. J. Rogerson, P. Mkundika, and M. K. Kanjalal, "Diagnosis of Plasmodium falciparum malaria at delivery: comparison of blood film preparation methods and of blood films with histology," Journal of Clinical Microbiology, vol. 41, no. 4, pp. 1370-1374, 2003.

[14] R. McGready, N. J. White, and F. Nosten, "Parasitological efficacy of antimalarials in the treatment and prevention of falciparum malaria in pregnancy 1998 to 2009: a systematic review," BJOG, vol. 118, no. 2, pp. 123-135, 2011.

[15] C. Lengeler, "Insecticide-treated bed nets and curtains for preventing malaria," Cochrane Database of Systematic Reviews, no. 2, p. CD000363, 2004.

[16] H. A. Vanga-Bosson, P. A. Coffie, S. Kanhon et al., "Coverage of intermittent prevention treatment with sulphadoxine-pyrimethamine among pregnant women and congenital malaria in Cote d'Ivoire," Malaria Journal, vol. 10, article 105, 2011.

[17] R. McGready, S. O. Tan, E. A. Ashley et al., "A randomised controlled trial of artemether-lumefantrine versus artesunate for uncomplicated plasmodium falciparum treatment in pregnancy," PLoS Medicine, vol. 5, no. 12, pp. 1699-1715, 2008.

[18] WHO, Guidelines for the Treatment of Malaria, World Health Organization, Geneva, Switzerland, 2nd edition, 2010, http://whqlibdoc.who.int/publications/2010/9789241547925_ eng.pdf.

[19] T. H. Holtz, S. P. Kachur, J. M. Roberts et al., "Use of antenatal care services and intermittent preventive treatment for malaria among pregnant women in Blantyre District, Malawi," Tropical Medicine and International Health, vol. 9, no. 1, pp. 77-82, 2004.

[20] E. N. Takem, E. A. Achidi, and P. M. Ndumbe, "Use of intermittent preventive treatment for malaria by pregnant women in Buea, Cameroon," Acta Tropica, vol. 112, no. 1, pp. 54-58, 2009.

[21] C. J. Uneke, "Diagnosis of Plasmoduim falciparum malaria in pregnancy in sub-Saharan Africa: the challenges and public health implications," Parasitology Research, vol. 102, no. 3, pp. 333-342, 2008.

[22] A. Mayor, E. Serra-Casas, A. Bardaji et al., "Sub-microscopic infections and long-term recrudescence of Plasmodium falciparum in Mozambican pregnant women," Malaria Journal, vol. 9, article 8, 2009.

[23] L. C. Okell, A. C. Ghani, E. Lyons, and C. J. Drakeley, "Submicroscopic infection in plasmodium falciparum-endemic populations: a systematic review and meta-analysis," Journal of Infectious Diseases, vol. 200, no. 10, pp. 1509-1517, 2009.

[24] L. M. Singer, R. D. Newman, A. Diarra et al., "Evaluation of a malaria rapid diagnostic test for assessing the burden of malaria during pregnancy," The American Journal of Tropical Medicine and Hygiene, vol. 70, no. 5, pp. 481-485, 2004.

[25] N. Singh, A. Saxena, S. B. Awadhia, R. Shrivastava, and M. P. Singh, "Evaluation of a rapid diagnostic test for assessing the burden of malaria at delivery in India," The American Journal of Tropical Medicine and Hygiene, vol. 73, no. 5, pp. 855-858, 2005.

[26] A. M. Rantala, S. M. Taylor, P. A. Trottman et al., "Comparison of real-time PCR and microscopy for malaria parasite detection in Malawian pregnant women," Malaria Journal, vol. 9, no. 1, article 269, 2010.

[27] T. Hanscheid and M. P. Grobusch, "How useful is PCR in the diagnosis of malaria?" Trends in Parasitology, vol. 18, no. 9, pp. 395-398, 2002.
[28] M. I. Masanja, M. McMorrow, E. Kahigwa, S. P. Kachur, and P. D. McElroy, "Health workers' use of malaria rapid diagnostic tests (RDTs) to guide clinical decision making in rural dispensaries, Tanzania," The American Journal of Tropical Medicine and Hygiene, vol. 83, no. 6, pp. 1238-1241, 2010.

[29] A. Bardaji, B. Sigauque, L. Bruni et al., "Clinical malaria in African pregnant women," Malaria Journal, vol. 7, article 27, 2008. 


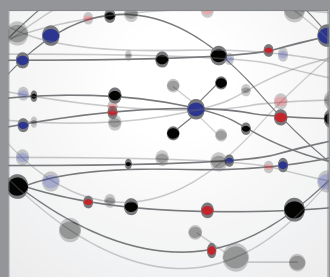

The Scientific World Journal
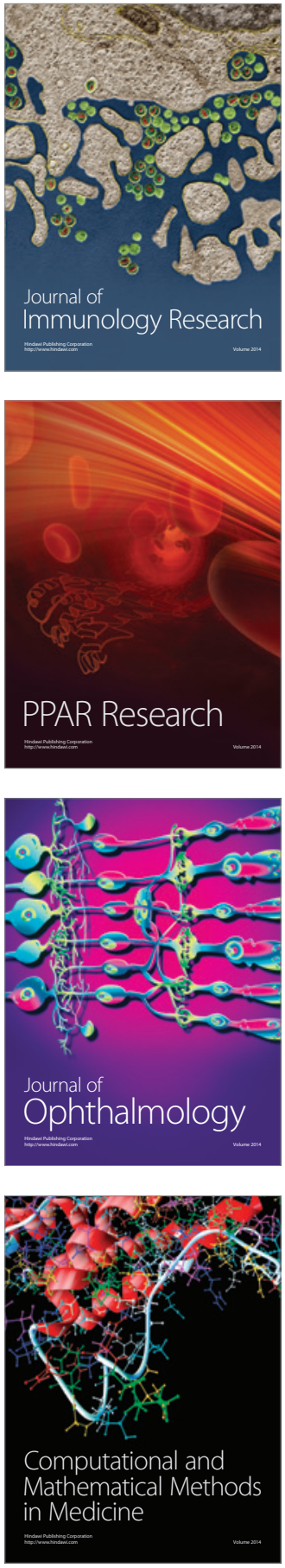

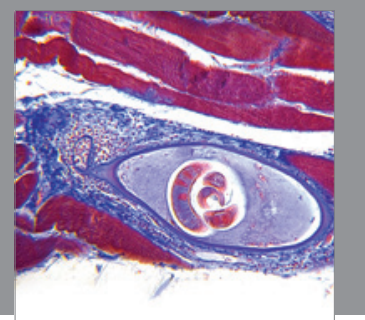

Gastroenterology

Research and Practice
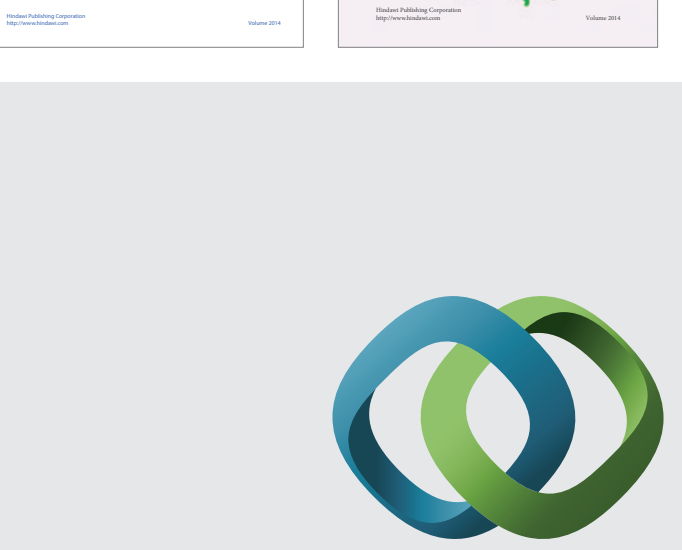

\section{Hindawi}

Submit your manuscripts at

http://www.hindawi.com
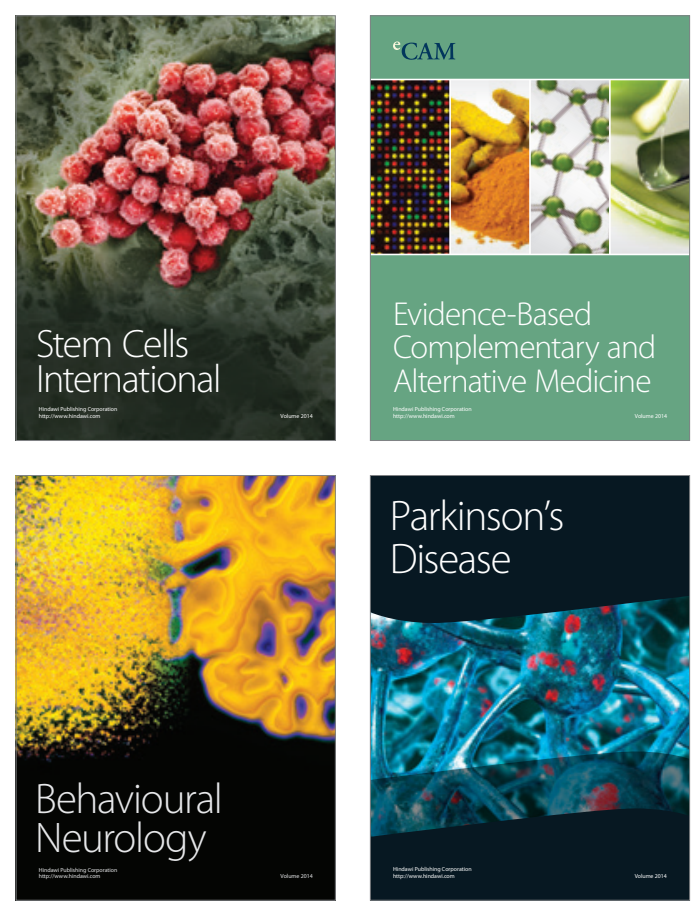

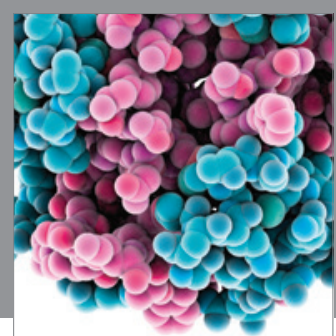

Journal of
Diabetes Research

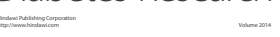

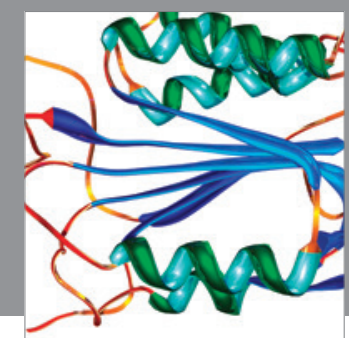

Disease Markers
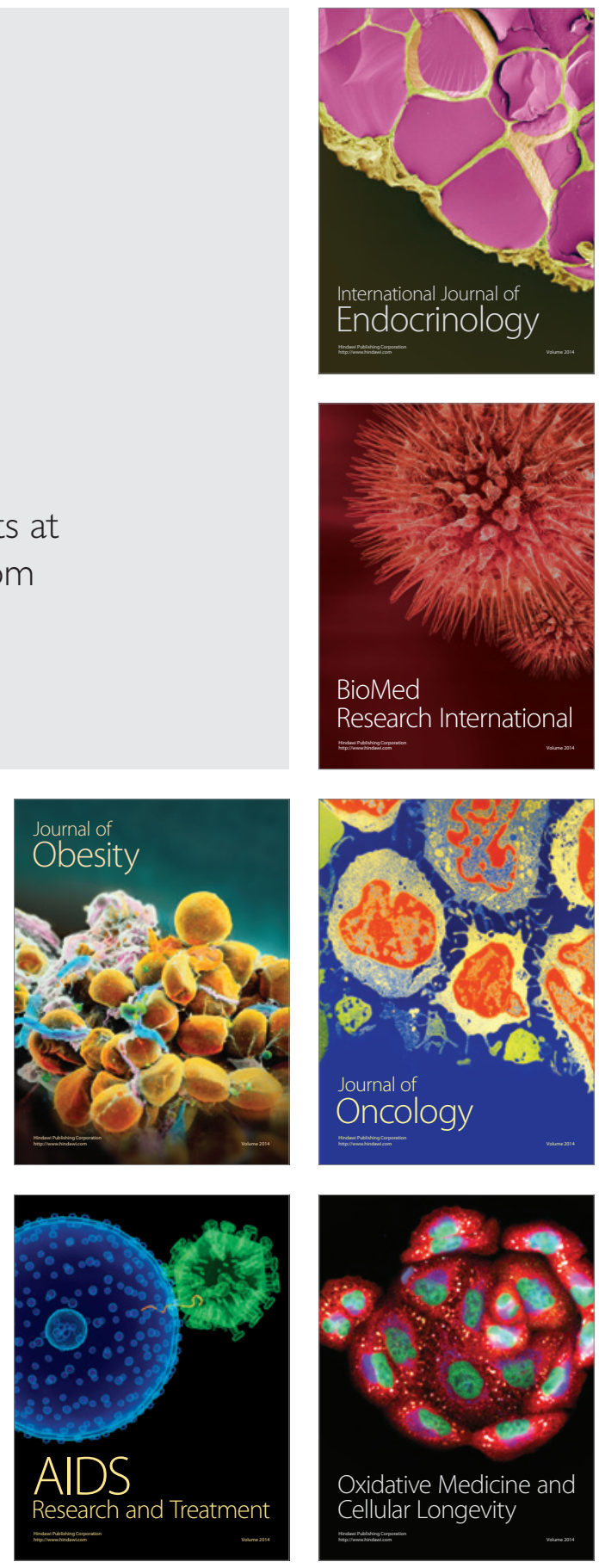